\title{
Dalibor Fronček
}

Graphs with a given edge neighbourhood

Czechoslovak Mathematical Journal, Vol. 39 (1989), No. 4, 627-630

Persistent URL: http://dml.cz/dmlcz/102338

\section{Terms of use:}

(C) Institute of Mathematics AS CR, 1989

Institute of Mathematics of the Czech Academy of Sciences provides access to digitized documents strictly for personal use. Each copy of any part of this document must contain these Terms of use.

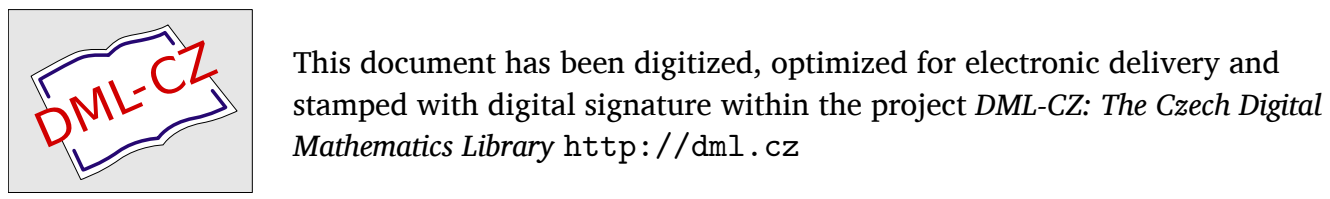




\title{
GRAPHS WITH A GIVEN EDGE NEIGHBOURHOOD
}

\author{
DALIBOR FronČEK, Ostrava
}

(Received April 21, 1987)

\section{INTRODUCTION}

All graphs considered in this paper are connected finite graphs without loops and multiple edges.

If the edge $e \in G$ joins the vertices $x, y$ then denote by $N_{G}(e)$ or $N_{G}(x, y)$ the subgraph of the graph $G$ induced by the set of all vertices adjacent to at least one of the vertices $x, y$ (except the vertices $x, y$ ). Analogously, denote by $N_{G}(x)$ the subgraph of $G$ induced by the set of all vertices adjacent to $x$.

A given graph $H$ is called edge-realizable or shortly e-realizable (vertex-realizable or $v$-realizable $)$ if there exists a graph $G$ in which the neighbourhood $N_{G}(e)$ of every edge $e\left(N_{G}(x)\right.$ of every vertex $\left.x\right)$ is isomorphic to $H$; in such a case $G$ is called an $e$-realization (a v-realization) of $H$. The set of all $e$-realizations ( $v$-realizations) of $H$ is denoted by $\mathscr{R}_{e}(H)\left(\mathscr{R}_{v}(H)\right)$.

The notion of $v$-realizable graphs was introduced by A. A. Zykov [4] and many authors have studied the properties of some families of these graphs. B. Zelinka [3] introduced the notion of $e$-realizable graphs and showed some families of them.

In this article some generalizations of the results of [3] are given.

\section{1. $e$-REALIZATIONS OF THE COMPLETE MULTIPARTITE GRAPHS}

Theorem A (Zelinka [3]). The complete bipartite graph $K_{n, m}$ is e-realizable.

A similar proposition for $v$-realizable graphs was suggested by B. Alspach and observed also by J. Doyen, X. Hubaud and M. Reynaert (see [2]).

Theorem B ([2]). The complete multipartite graph $K_{n_{1}, n_{2}, \ldots, n_{k}}$ is not v-realizable unless $n_{1}=n_{2}=\ldots=n_{k}$.

The next generalization of Theorem A is an analogue of Theorem B for $e$-realizable graphs.

Theorem 1. The complete multipartite graph $K_{n_{1}, n_{2}, \ldots, n_{k}}$ is e-realizable if and only if

$$
n_{1}+1=n_{2}+1=n_{3}=\ldots=n_{k} \text {. }
$$


To prove this theorem, we will use the following

Lemma 1.1. Let $G$ be isomorphic to $K_{n_{1}, n_{2}, \ldots, n_{k}}(k \geqq 3)$. Then $N_{G}(e) \simeq N_{G}(f)$ for each pair of edges $e, f$ of $G$ if and only if $n_{1}=n_{2}=\ldots=n_{k}$.

Proof of this lemma is simple and can be left to the reader.

Proof of Theorem 1. $(\Leftarrow)$ If $G \simeq K_{n, n, \ldots, n}$ then $N_{G}(e) \simeq K_{n-1, n-1, n, \ldots, n}$.

$(\Rightarrow)$ Let $G$ be an $e$-realization of $K_{n_{1}, n_{2}, \ldots, n_{k}}$. Then $N_{G}\left(y_{1}, y_{2}\right) \simeq K_{n_{1}, n_{2}, \ldots, n_{k}}$ for each pair of the adjacent vertices $y_{1}, y_{2}$. Denote the parts of $N_{G}\left(y_{1}, y_{2}\right)$ by $P_{1}, P_{2}, \ldots$ $\ldots, P_{k}$ and the vertices of $P_{i}$ by $x_{1}^{i}, x_{2}^{i}, \ldots, x_{n_{i}}^{i}$ for each $i=1,2, \ldots, k$. Without losing generality we can suppose that

$$
n_{1} \leqq n_{2} \leqq \ldots \leqq n_{k}
$$

Now explore the neighbourhood of the edge $x_{i}^{1}, x_{j}^{2}$. As $G \in \mathscr{R}_{e}\left(K_{n_{1}, n_{2}, \ldots, n_{k}}\right)$ hence

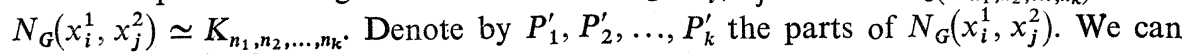
see that $N_{G}\left(x_{i}^{1}, x_{j}^{2}\right)$ contains the vertices $y_{1}, y_{2}$ and the graph $F \simeq K_{n_{1}-1, n_{2}-1, n_{3}, \ldots, n_{k}}$ with the parts $P_{1}-x_{i}^{1}, P_{2}-x_{j}^{2}, P_{3}, \ldots, P_{k}$. Since the vertices $y_{1}, y_{2}$ are adjacent, each part of $N_{G}\left(x_{i}^{1}, x_{j}^{2}\right)$ can contain at most one of these vertices. It follows from (1) that $P_{3}^{\prime}=P_{3}, P_{4}^{\prime}=P_{4} \ldots, P_{k}^{\prime}=P_{k}$ and each of the parts $P_{1}^{\prime}, P_{2}^{\prime}$ contains exactly one of the vertices $y_{1}, y_{2}$. Without loss of generality we can suppose that $P_{1}^{\prime}=$ $=P_{1}-x_{i}^{1}+y_{1}$ and $P_{2}^{\prime}=P_{2}-x_{j}^{2}+y_{2}$. Therefore the vertex $y_{1}$ is adjacent to $x_{j}^{2}$ and to all vertices of the parts $P_{2}^{\prime}, P_{3}^{\prime}, \ldots, P_{k}^{\prime}$. Analogously, $y_{2}$ is adjacent to $x_{i}^{1}$ and to all vertices of the parts $P_{1}^{\prime}, P_{3}^{\prime}, P_{4}^{\prime}, \ldots, P_{k}^{\prime}$. Thus $G$ contains a subgraph isomorphic to $K_{n_{1}+1, n_{2}+1, n_{3}, \ldots, n_{k}}=K$.

As $N_{G}\left(y_{1}, y_{2}\right) \simeq K_{n_{1}, n_{2}, \ldots, n_{k}}$, the number of its vertices is $\left|N_{G}\left(y_{1}, y_{2}\right)\right|=n_{1}+$ $+n_{2}+\ldots+n_{k}=n_{0}$. Since $G \in \mathscr{R}_{e}\left(K_{n_{1}, n_{2}, \ldots, n_{k}}\right)$, the equality $\left|N_{G}(f)\right|=n_{0}$ holds for every edge $f$ of $G$. On the other hand, $\left|N_{K}(f)\right|=n_{0}$ and hence $G=K$.

Under Lemma $1.1 G \simeq K_{n, n, \ldots, n}$ and this yields $n_{1}+1=n_{2}+1=n_{3}=\ldots=n_{k}$.

\section{2. $e$-REALIZATIONS OF THE CYCLES}

M. Brown and R. Connelly proved the following

Theorem C ([1]). All cycles are vertex-realizable.

In his article [3] Zelinka has shown that the cycles $C_{3}, C_{4}, C_{6}, C_{8}$ are $e$-realizable and $C_{.5}$ is not $e$-realizable.

The next theorem is a generalization of this result.

Theorem 2. The cycles $C_{2 n+1}$ are not e-realizable, with the single exception of $C_{3}$. To prove this theorem we need the following

Lemma 2.1. Let the graph $H=K_{4}-e$ be a subgraph of $G$. Then $G$ is not an e-realization of $C_{2 n+1}$ for $n>1$.

Proof. Let $K_{4}$ be the complete graph with vertices $y_{1}, y_{2}, y_{3}, y_{4}$ and let $e^{2}=y_{3}, y_{4}$. Suppose that $H=K_{4}-e$ is a subgraph of $G$. Let $N_{G}\left(y_{1}, y_{2}\right)$ be isomorphic to $C_{2 n+1}$ 
with the vertices $x_{0}, x_{1}, x_{2}, \ldots, x_{2 n}$. Without loss of generality we can identify $y_{3}$ with $x_{0}$ and $y_{4}$ with $x_{j}$. It is evident that $x_{0}$ is not adjacent to $x_{j}-$ in the opposite case $N_{G}\left(y_{1}, x_{i}\right)$ (or $N_{G}\left(y_{2}, x_{i}\right)$ ) for any $i \neq 0, j$ contains the cycle $C_{3}$ induced by the vertices $y_{2}, x_{0}, x_{j}\left(y_{1}, x_{0}, x_{j}\right)$. Thus $2 \leqq j \leqq 2 n-1$.

Now suppose that there exists an edge $x_{i}, x_{i+1}(i \neq 0, j-1, j, 2 n)$ such that $x_{i}$ is adjacent to $y_{1}$ and $x_{i+1}$ is adjacent to $y_{2}$. Then $N_{G}\left(x_{i+1}, y_{2}\right)$ contains the subgraph $K_{1,3}$ with the vertices $y_{1}, x_{c}, x_{j}, x_{i}$, which is a contradiction. Analogously, if $x_{i}$ is adjacent to $y_{2}$ and $x_{i+1}$ is adjacent to $y_{1}$ then $N_{G}\left(x_{i+1}, y_{1}\right)$ contains $K_{1,3}$ with the vertices $y_{2}, x_{0}, x_{j}, x_{i}$. Thus all the vertices $x_{1}, x_{2}, \ldots, x_{j-1}$ have to be adjacent to exactly one vertex of the pair $y_{1}, y_{2}$. Without losing generality we can suppose that it is the vertex $y_{1}$.

Analogously, all the vertices $x_{j+1}, x_{j+2}, \ldots, x_{2 n}$ are adjacent to exactly one vertex $y$ of the pair $y_{1}, y_{2}$.

Now just one of the following cases occurs:

(i) $y=y_{1}$. Then $N_{G}\left(y_{1}, x_{0}\right)$ contains a subgraph $K_{1,3}$ with the vertices $x_{j-1}, x_{j}, y_{2}$ and hence $G \notin \mathscr{R}_{e}\left(C_{2 n+1}\right)$.

(ii) $y=y_{2}$. Then $N_{G}\left(y_{2}, x_{j}\right)$ contains the path $x_{j+1}, x_{j+2}, \ldots, x_{2 n}, x_{0}, y_{1}, x_{j-1}$. If $G \in \mathscr{R}_{e}\left(C_{2 n+1}\right)$ then $N_{G}\left(y_{2}, x_{j}\right) \simeq C_{2 n+1}$ with the vertices $x_{0}, y_{1}, x_{j-1}, z_{3}, z_{4}, \ldots$ $\ldots, z_{j}, x_{j+1}, x_{j+2}, \ldots, x_{2 n}$. Suppose that $z_{i}=x_{r}$ for any $i \in\{3,4, \ldots, j\}, r \in$ $\in\{1,2, \ldots, j-2\}$. As $G \in \mathscr{R}_{e}\left(C_{2 n+1}\right)$ hence either $x_{r}$ is adjacent to $x_{j}$ (and $\left.N_{G}\left(y_{1}, y_{2}\right) \neq C_{2 n+1}\right)$, or $x_{r}$ is adjacent to $y_{2}$ (and $N_{G}\left(x_{r}, y_{2}\right)$ contains $K_{1,3}$ - see above). Thus $z_{i} \neq x_{r}$.

Hence $N_{G}\left(y_{1}, x_{j}\right)$ contains the cycle $C_{2 j}$ with $2 j$ vertices $x_{0}, x_{1}, \ldots, x_{j-1}$, $z_{3}, z_{4}, \ldots, z_{j}, x_{j+1}, y_{2}$, which is a contradiction. Thus $G \notin \mathscr{R}_{e}\left(C_{2 n+1}\right)$.

Now we are able to prove Theorem 2.

Proof of Theorem 2. Let $e=y_{1}, y_{2}$ be any edge of $G$ and let $\left\{x_{0}, x_{1}, \ldots, x_{2 n}\right\}$ be the vertex set of $N_{G}(e) \simeq C_{2 n+1}$. If there exists a vertex $x_{i}$ which is adjacent to both vertices $y_{1}$ and $y_{2}$ then the graph $G$ contains the graph $H$ from Lemma 2.1 with the vertex set $\left\{x_{i}, x_{i+1}, y_{1}, y_{2}\right\}$, and hence $G \notin \mathscr{R}_{e}\left(C_{2 n+1}\right)$.

Thus each vertex $x_{i}$ is adjacent to exactly one vertex of the pair $y_{1}, y_{2}$. Since $C_{2 n+1}$ contains an odd number of vertices, there exists a triangle induced by the vertices $y_{1}, x_{i}, x_{i+1}$ (or $y_{2}, x_{i}, x_{i+1}$ ). Without losing generality we can suppose that it is the triangle $y_{1}, x_{0}, x_{1}$. Then $x_{2}$ is adjacent to $y_{2}$ (in the opposite case the vertices $x_{0}, x_{1}, x_{2}, y_{1}$ induce the graph $H$ ) and $x_{3}$ has to be also adjacent to $y_{2}$ (in the opposite case $N_{G}\left(x_{1}, x_{2}\right)$ contains the subgraph $K_{1,3}$ with the vertices $y_{1}, y_{2}, x_{0}, x_{3}$, which is a contradiction). Hence the vertices $x_{4 k}, x_{4 k+1}$ are adjacent to $y_{1}$ and the vertices $x_{4 k+2}, x_{4 k+3}$ are adjacent to $y_{2}$. If $n$ is an even number then $x_{2 n}$ is adjacent to $y_{1}$ and $G$ contains the subgraph $H$ with the vertices $x_{2 n}, x_{0}, y_{1}, y_{2}$, which is a contradiction. If $n$ is an odd number then $x_{2 n}$ is adjacent to $y_{2}$. In this case $x_{2 n-1}$ is adjacent to $y_{1}$ and thus $N_{G}\left(x_{2 n}, x_{0}\right)$ contains the subgraph $K_{1,3}$ with tie vertices $y_{1}, y_{2}, x_{2 n-1}, x_{1}$, which is also a contradiction. Therefore $G \notin \mathscr{R}_{e}\left(C_{2 n+1}\right)$ and hence $C_{2 n+1}$ is not edge-realizable. 
On the other hand, e-realizability of the even cycles was proved by R. Nedela [5]. Theorem D (Nedela). The cycles $C_{2 n}$ are e-realizable for each $n \geqq 2$.

From this Theorem and our Theorem 2 we obtain the following

Corollary. $A$ cycle $C_{n}$ is e-realizable if and only if $n$ is an even number or $n=3$.

\section{References}

[1] M. Brown, R. Connelly: On graphs with a constant link. In: Proof techniques in graph theory (F. Harary ed.), Academic press 1969.

[2] J. Doyen, $X$. Hubaud, M. Reynaert: Finite graphs with isomorphic neighbourhood. In: Colloque CNRS. Problèmes combinatoires et théorie des graphes, Orsay 1976.

[3] B. Zelinka: Edge neighbourhood graphs. Czech. Math. Journ. 36 (111), 1986, 44-47.

[4] A. A. Zykov: Problem 30. In: Theory of graphs and its applications. Proc. Symp. Smolenice 1963 (ed. M. Fiedler), Prague 1964, 164-165.

[5] R. Nedela: Graphs which are edge-locally $C_{n}$. Submitted to Czech. Math. Journ.

Author's address: 70833 Ostrava, tř. Vítězného února, Czechoslovakia (Vysoká škola báňská). 